Gut, 1988, 29, 969-973

\title{
Outcome of colectomy for severe idiopathic constipation
}

\author{
M A KAMM, P R HAWLEY, AND J E LENNARD-JONES \\ From St Marks Hospital, London
}

SUMMARY Severe idiopathic constipation affects predominantly young women. We have evaluated the results of colectomy in 44 women with a normal diameter colon and with a median bowel frequency of once per four weeks. The operations were done in one hospital over a 15 year period. After operation 22 patients had normal bowel frequency, 17 diarrhoea (greater than three bowel actions/day), and five persistent or recurrent constipation (interval between bowel actions at least three days). Many patients continued to experience abdominal pain (98\% preoperatively $v \mathbf{7 1 \%}$ postoperatively); straining and laxative use were still common after surgery. Ten patients needed psychiatric treatment for severe psychological disorders. The preoperative abnormalities of paradoxical contraction of the pelvic floor during straining and impaired rectal evacuation of a water filled balloon did not correlate with the outcome.

Severe constipation can be a disabling condition which predominantly affects women of childbearing age.' The major symptoms, which include infrequent and difficult defecation, abdominal pain and bloating, ' are often resistant to medical therapy or dietary manipulation. In 1908 Arbuthnot Lane described the results of colectomy in 39 patients. ${ }^{2}$ Although some of his patients had other colonic or non-colonic conditions, most were suffering from severe constipation with macroscopically normal colons. Colectomy remains an accepted procedure when symptoms dominate a patient's life and medical therapy has failed.

With the development of techniques which enable the anorectal expulsion mechanism to be more closely studied, it has become apparent that many of these patients have an abnormality of pelvic floor function and rectal evacuation. ${ }^{3-5}$ Recent surgical emphasis has therefore focussed on the pelvic floor, and the value of colectomy in patients with incoordinate pelvic floor musculature has been questioned."

We have evaluated the results of colectomy in all the patients with a macroscopically normal colon who

Address for correspondence: Dr M Kamm. St Marks Hospital, City Road. London ECIV 2PS.

Received for publication 22 January 1988 have been operated upon for constipation over a 15 year period in one hospital. Attention has been paid not only to the functional and symptomatic results of surgery but also to the predictive value of preoperative anorectal physiology studies with regard to outcome.

Methods .

PATIENTS

An analysis of hospital records showed that 44 patients with a normal diameter rectum and colon on barium enema underwent total colectomy for severe idiopathic constipation between 1972 and February 1987. All patients have been included in this study. Hirschsprung's disease was excluded by the presence of the rectoanal inhibitory reflex, or a full thickness rectal biopsy, or both. The presence of normal ganglia was also confirmed in all cases in the resected specimens.

Patients with radiologically defined idiopathic megarectum or megacolon have not been included.

In addition to examination of the 44 records, 32 of the patients were interviewed (by MK) postoperatively to obtain further information. Data on some of the postoperative symptoms in the remaining 12 patients were not available and could not be included in the study. 
Table 1 Clinicalpresentation

\begin{tabular}{ll}
\hline Patients $(n)$ & 4 \\
Statedinterval between spontaneous bowel actions \\
- median & 4 weeks \\
- range & 1 day-12 wecks \\
Abdominal pain & $43(98 \%)$ \\
Straining on most occasions & $42(95 \%)$ \\
Laxative use preoperative & $39(88 \%)$ \\
Bloating & $42(95 \%)$ \\
\hline
\end{tabular}

Clinical. Details (Table 1)

All the patients were women. The mean age at operation was 34 years, with a range of 18 to 74 . The age of onset of constipation varied from infancy to 67 years, in 32 patients the onset was in the first two decades. The onset of constipation was not associated with any precipitating event in 39 patients. In the other five patients, constipation was precipitated in one patient by rectopexy for rectal prolapse (the oldest patient, aged 74, who underwent rectopexy at the age of 67), childbirth in two (no obstetric tear occurred in either), immobilisation after a car accident (there was no abdominal or pelvic trauma), and a fall in which a patient's perineum landed on a metal bar (blunt trauma).

Only one patient of the 44 had a stated preoperative spontaneous bowel frequency of greater than once per week; the median stated spontaneous bowel frequency was once per four weeks, and the greatest interval was 12 weeks. Abdominal pain was a prominent symptom and was present in 43 patients $(98 \%)$ preoperatively. Forty two patients $(95 \%)$ strained on most occasions at stool, and $42(95 \%)$ also complained of abdominal bloating. All the patients had taken laxatives at some stage; and $39(88 \%)$ were using them up until the time of surgery.

All patients had tried numerous laxatives and increased dietary fibre in the past without benefit. No patient had an alternating bowel habit or experienced episodes of diarrhoea. Previous surgery was common. Five patients had undergone rectopexy with no improvement in their constipation. One patient had had a temporary ileostomy. Three had had a previous partial colonic resection, and two had undergone an anal stretch. There was also a high incidence of other operations; 21 patients had a total of 41 previous non-colonic or nonanorectal operations, including hysterectomy in 11 patients, 10 appendicectomies, laparotomy for pain in four patients, and ovarian cystectomies in two patients.

Assessment for surgery usually included at least one interview with either the medical social worker or psychiatrist, but six patients had previous psychiatric treatment independent of this assessment. One patient had had anorexia nervosa, one hysterical weakness, and one serious behavioural problems. Two patients had been seriously depressed previously, and another patient had taken a drug overdose. Because pain is a prominent feature of this condition, several patients required strong analgesia; one patient - for example - had had 12 hospital admissions elsewhere for pain severe enough to be relieved with narcotic analgesics, in the six months before surgery.

The following tests were included in the preoperative assessment as they came into common clinical use:

WHOLEGUT TRANSIT STUDIES

Whole gut transit time was assessed using the method of Hinton et al. ${ }^{\times}$Twenty radioopaque shapes were swallowed and a plain abdominal radiograph taken five days later. Thirty five of $36(97 \%)$ had prolonged transit, as shown by the retention of more than four shapes.

BALLOON EXPULSION

In a test of rectal evacuation patients lay in the left lateral position." A rubber balloon attached to a $4 \mathrm{~mm}$ diameter plastic catheter was lubricated and inserted into the rectal ampulla. The balloon was filled with $50 \mathrm{ml}$ water and the patient asked to strain and expel the balloon. Twenty one of 29 patients $(72 \%)$ were unable to expel the balloon.

\section{PUBORECTALIS ELECTROMYOGRAPHY}

To assess pelvic floor striated muscle activity during straining (with the patient still in the left lateral position) a concentric electromyography needle was inserted $12 \mathrm{~mm}$ posterior to the anal margin in the midline and directed slightly anteriorly till activity was recorded from the puborectalis muscle." "I" The patient was then asked to strain, and the change in muscle activity was recorded as increased, unchanged, or decreased. Thirteen of $20(65 \%)$ showed either an increase or no change in muscle activity, compared with the normal decrease in activity.

\section{SURGERY}

Eleven patients had a caecorectal anastomosis, while more recently 33 patients had an ileorectal anastomosis. The anastomosis was at the level of the sacral promontory.

\section{Results}

All the resected colons were macroscopically normal and of normal diameter. Routine hamatoxylin and eosin staining of the resected colons showed melanosis coli in 16 patients and normal histology in 28 
Table 2 Symptomatic results aftersurgery

\begin{tabular}{lc}
\hline Normal bowel frequency & $22(50 \%)$ \\
Diarrhoea ( $>3$ bowel actions per day) & $17(39 \%)$ \\
Constipation (bowel actions<every 3 days) & $5(11 \%)$ \\
Incontinence & $6(14 \%)$ \\
Abdominal pain & $30 / 42^{*}(71 \%)$ \\
Straining & $13 / 39 *(33 \%)$ \\
Bloating & $15 / 33 *(45 \%)$ \\
\hline
\end{tabular}

*Figures quoted for those in whom follow up available.

patients. The nerve plexuses and muscle coats appeared normal in all specimens on $\mathrm{H} \& \mathrm{E}$ staining.

The mean follow up was three years two months, with a range of three months to 14 years.

BOWEL FREQUENCY (Table 2)

The operation was very successful in increasing the bowel frequency in most patients. Twenty two patients had a normal bowel frequency postoperatively, but 17 patients had diarrhoea (bowel frequency greater than three times per day) and five had persistent or recurrent constipation (period between spontaneous bowel actions greater than three days). Incontinence, usually mild soiling, was experienced by six patients.

Twelve of $40(30 \%)$ patients still used laxatives on some occasions postoperatively.

\section{OTHER SYMPTOMS}

Thirty of $42(71 \%)$ patients still experienced abdominal pain postoperatively, although most thought it had improved. In a minority the pain remained severe, and occasionally this was a source of diagnostic difficulty when patients presented with symptoms suggestive of bowel obstruction.

Fifteen of 33 patients (45\%) still experienced bloating postoperatively, although again this was often improved. Thirteen of $39(33 \%)$ still strained at stool. Four patients have had serious psychiatric problems since the operation. One patient was admitted to hospital because of depression and another committed suicide. A third patient took a drug overdose. A fourth patient who complained of diarrhoea had proven surreptitious laxative abuse.

\section{FURTHER SURGERY}

Seventeen patients have had further surgery subsequent to their colectomy, and this was more likely with increasing time. Eight patients have had a laparotomy for suspected bowel obstruction, with freeing of adhesions. These were done in other hospitals, and it was not possible to establish in how many of these cases the diagnosis was confirmed at laparotomy.

Six patients have had a terminal ileostomy created, three for persistent constipation, two for diarrhoea, and one for diarrhoea together with incontinence. Three patients have undergone puborectalis division for persistent constipation, without relief, and one patient has had three puborectalis divisions for constipation plus a Dolorme procedure for prolapse.

\section{ANORECTAL PHYSIOLOGY AND OUTCOME (Table 3)}

\section{Balloon expulsion}

Patients who could not expel a balloon preoperatively had a similar mean preoperative bowel frequency to those who could. The mean bowel frequency for both groups was similar postoperatively. In addition, the proportion of patients with diarrhoea, normal bowel frequency, and constipation was similar in the two groups.

\section{Puborectalis electromyography}

The preoperative bowel frequency for those with paradoxical muscle contraction was similar to those who relaxed their pelvic floor on straining. The mean bowel frequency after surgery was improved to a similar degree in both groups. The proportion with postoperative diarrhoea, normal bowel frequency and constipation was similar for the two groups. Nine of 13 patients with preoperative paradox had a bowel action once per day or more frequently after the operation.

\section{Long versus short term outcome (Table 4)}

To determine whether some of the unwanted effects and complications of surgery resolved with time, and to determine whether the benefits of surgery were maintained, the patients have been divided into those with follow up less than or equal to two years (20) patients, mean follow up one year) and those with follow up more than two years ( 24 patients, mean follow up five years and one month). The two groups had similar preoperative characteristics, with similar preoperative mean spontaneous bowel frequency, laxative use, and incidence of pain.

The mean postoperative spontaneous bowel frequency was similar for both groups, suggesting that the benefit of surgery tends to be maintained. There was, however, an increased use of laxatives in the long follow up group, but the number with spontaneous infrequent bowel actions (less than every three days) was similar.

The proportion of patients with diarrhoea was smaller in the long follow up group, suggesting that in some patients this symptom may improve with time, but the difference was not statistically significant.

The incidence of pain in the two groups was similar suggesting that postoperative pain tends to persist. 


\begin{tabular}{|c|c|c|c|c|c|c|}
\hline & \multirow[b]{2}{*}{$\begin{array}{l}\text { Patients } \\
\text { (n) }\end{array}$} & \multicolumn{2}{|c|}{$\begin{array}{l}\text { Mean stated interval between } \\
\text { spontaneous bowel actions }\end{array}$} & \multicolumn{3}{|c|}{ Post op bowel frequency } \\
\hline & & $\begin{array}{l}\text { Preop) } \\
\text { (weeks) }\end{array}$ & $\begin{array}{l}\text { Postop } \\
\text { (days) }\end{array}$ & Constipation & Normal & Diarrhoea \\
\hline Puborectalis-paradox or nochange & 13 & $4 \cdot 8$ & $3 \cdot 5$ & 4 & 5 & 4 \\
\hline Puborectalis relaxation & 7 & $4 \cdot 5$ & 0.9 & () & 5 & 2 \\
\hline Balloon expulsion - able & 8 & 3.9 & $1 \cdot 4$ & 1 & 6 & 1 \\
\hline Balloon expulsion - unable & 21 & $4 \cdot 9$ & $2 \cdot()$ & 3 & 10 & 8 \\
\hline
\end{tabular}

\section{Discussion}

When Arbuthnot Lane first described the results of colectomy for idiopathic constipation he believed the outcome was universally satisfactory." This study shows that there are problems with colectomy and extends our observations on a smaller series reported from this hospital previously." The results in this relatively large series of 44 patients give a different impression from the other reports in the literature. Klatt reported "2 a good outcome in nine patients who underwent colectomy with ileal anastomosis to the lower sigmoid. Mean follow up was two years and no patient required laxatives postoperatively. Keighley and Shouler ${ }^{7}$ also reported good results in nine of 10 patients with slow transit who underwent colectomy. Beck et al" reported the results of colectomy in 14 patients, eight of whom had had a previous hysterectomy. The patients were thought to suffer from colonic inertia, although only two had transit studies. Follow up for a mean of one year and two months in 11 of the patients revealed a satisfactory outcome, with bowel frequency improved from a mean of once per 10 days to approximately twice per day.

Table 4 Short versus long term results

\begin{tabular}{|c|c|c|}
\hline Follow up (years) & $\leqslant 2 \cdot()$ & $>2 \cdot()$ \\
\hline n & 20 & 24 \\
\hline Mean follow up (years) & $1 \cdot()$ & $5 \cdot 1$ \\
\hline Type of anastomosis - caecorectal & 4 & 7 \\
\hline - ileorectal & 16 & 17 \\
\hline \multicolumn{3}{|l|}{ Mean interval between spontaneous } \\
\hline bowel actions - preop & 3.6 weeks & $5 \cdot 2$ weeks \\
\hline- postop & 1.4 days & $1 \cdot 8$ days \\
\hline Pain-preop & $20 / 20(100 \%)$ & $23 / 24(96 \%)$ \\
\hline - postop & $13 / 18(72 \%)$ & $17 / 24(71 \%)$ \\
\hline Laxative use-preop & $17 / 20(85 \%)$ & $17 / 19(89 \%)$ \\
\hline$\quad-$ postop & $3 / 20(15 \%)$ & $9 / 20(45 \%)$ \\
\hline Constipation (interval $>3$ days) & 2 & 3 \\
\hline Diarrhoea (>3/day) & 10 & 7 \\
\hline Further surgery & 3 & 14 \\
\hline
\end{tabular}

For pain and laxative use, figures quoted for those in whom followup is available.
Analysis of our results (Table 4) reveals that the satisfactory bowel frequency produced by surgery in half the patients was maintained. Diarrhoea was, however, troublesome and persisted in almost $30 \%$ of patients, and constipation persisted in about $10 \%$ of patients. Nearly half the patients with long follow up were using laxatives at least intermittently, perhaps related to recurrent anorectal problems with defecation straining, rather than infrequent defecation. The high incidence $(71 \%)$ of postoperative pain did not change significantly with time. The incidence of further abdominal surgery was clearly related to the duration of follow up; a few patients were treated by another operation for disabling diarrhoea or constipation and one fifth had a laparotomy for presumed bowel obstruction.

With the introduction of methods which enable the evaluation of rectal evacuation and pelvic floor function, it has become popular to divide patients with severe constipation into those with slow colonic transit and a presumed colonic abnormality, and those with a predominantly pelvic floor problem."7 Some centres are reluctant to carry out colonic resection in those patients with features of abnormal evacuation, in the fear that these patients will have difficulty with rectal evacuation of even liquid stool." We correlated the postoperative outcome with a test of rectal evacuation and electromyographic evidence of inappropriate pelvic floor activity on straining. Failure to expel a rectal balloon or paradoxical contraction of the puborectalis muscle on straining did not predict a bad outcome in these patients treated by colectomy. Balloon expulsion is a model for rectal evacuation which is often abnormal in these patients, but it does not mimic normal defecation which involves the integration of many stimuli and muscle groups, including some higher than the rectal ampulla. Similarly, inappropriate puborectalis muscle activity may be only one aspect of a wider disturbance in constipation; it has been observed in other anorectal conditions and some normal individuals..$^{13}$

There is increasing recognition of a serious psycho- 
logical disorder in many of these patients ${ }^{1+15}$ and 10 of the patients in this series had a clear history of psychiatric illness either before or after the operation. We have been impressed by the high incidence of a disturbed background in many patients with this condition, as well as anxiety or depressive symptoms. When we asked 20 patients, similar to those described here, to complete formal psychiatric questionnaires the results showed little difference from normal or from controls with inflammatory bowel disease. ${ }^{14} \mathrm{~A}$ structured psychiatric interview, however, revealed a very high incidence of disturbed childhood or evidence of psychiatric disorder in the patients with constipation. ${ }^{14} \mathrm{~A}$ recent questionnaire based evaluation ${ }^{15}$ restricted to patients undergoing surgery (anorectal myectomy or colectomy) for severe constipation showed higher anxiety and depression scores than matched controls. In addition, those who had a good outcome with surgery could be predicted by their lower preoperative anxiety and depression scores. ${ }^{15}$ Patients' psychological state influences their symptom tolerance and may result in patients with the lowest threshold seeking surgery. Furthermore, this group may also tolerate the side effects of surgery least well. A preoperative psychosocial assessment is therefore essential.

Operations for idiopathic constipation other than total colectomy have yielded unpredictable results. Segmental colonic resection is usually followed by recurrent constipation. " Division of the puborectalis muscle, in those patients with inappropriate pelvic floor activity, has also not been successful. ${ }^{16}{ }^{17}$ In the belief that internal sphincter dysfunction may contribute to or cause constipation, two groups have reported good results after internal sphincter myectomy ${ }^{18}{ }^{19}$ Martelli et al $l^{18}$ reported good results with this operation, but the group of patients was heterogeneous, including some with Hirschsprung's disease. Yoshioka and Keighley ${ }^{19}$ also reported good results with this operation, and correlated a good outcome with an effective decrease in the resting anal pressure.

Total colectomy in patients with severe idiopathic constipation is usually effective in relieving the constipation, but there are frequently residual or new symptoms. An improvement in the surgical approach to this problem will depend on a better delineation of the physiological abnormality.

This study was supported by the St Marks Research Foundation.

\section{References}

1 Preston DM. Lennard-Jones JE. Severe chronic constipation of young women: idiopathic slow transit constipation'. Gut 1986, 27: 41-8.

2 Arbuthnot Lane $W$. The results of the operative treatment of chronic constipation. $B r M e d J$ 1908: 1: 126-30.

3 Preston DM, Lennard-Jones JE. Anismus in chronic constipation. Dig. Dis Sci 1985; 30: 41.3-8.

4 Read NW, Timms JM. Barfield LJ. Donnclly TC. Bannister JJ. Impairment of defaccation in young women with severe constipation. Gastroenterology 1986; 90: 53-60).

5 Shouler P, Keighley MRB. Changes in colorectal function in severe idiopathic constipation. Gastroenterology 1986; 90: 414-20.

6 Beck DE, Jagelman DG, Fazio VW. The surgery of idiopathic constipation. Gastroenterol Clin N Am 1987; 16: $143-56$.

7 Keighley MRB. Shouler P. Outlet syndrome: is there a surgical option? J R Soc Med 1984; 77: 559-63.

8 Hinton JM, Lennard-Jones JE, Young AC. A new method for studying gut transit times using radioopaque markers. Gut 1969; 10: 842-7.

9 Barnes PRH, Lennard-Jones JE. Balloon expulsion from the rectum in constipation of different types. Gut 1985; 26: 1049-52.

10 Turnbull GK, Lennard-Jones JE, Bartram CI. Failure of rectal expulsion as a cause of constipation: why fibre and laxatives sometimes fail. Lancet 1986; i: 767-9.

11 Preston DM, Hawley PR, Lennard-Jones JE, Todd IP. Results of colectomy for severe idiopathic constipation in women (Arbuthnot Lane's disease). Br J Surg 1984; 71: $547-52$.

12 Klatt GR. Role of subtotal colectomy in the treatment of incapacitating constipation. Am J Surg 1983; 145: 623-5.

13 Jones PN, Lubowski DZ. Henry MM, Swash M. Is paradoxical contraction of puborectalis muscle of functional importance? Dis Colon Rectum 1987; 30: 667-70.

14 Preston DM, Pfeffer JM, Lennard-Jones JE. Psychiatric assessment of patients with severe constipation [Abstract]. Gut 1984; 25: A582-3.

15 Fisher SE, Keighley MRB, Brecon K, Smart V, Andrews $\mathrm{H}$. Do patients with disordered defaccation have a primary personality disorder? [Abstract]. Gut 1987; 28: A 1373.

16 Barnes PRH, Hawley PR, Preston DM, Lennard-Jones $\mathrm{JE}$. Experience of posterior division of the puborectalis muscle in the management of chronic constipation. $\mathrm{Br} J$ Surg 1985; 72: 475-7.

17 Kamm MA. Hawley PR, Lennard-Jones JE. Experience of lateral puborectalis division for severe constipation [Abstract]. Gut 1987; 28: A 1363.

18 Martelli H, Devroede G, Arhan P, Duguay C. Mechanisms of idiopathic constipation: outlet obstruction. Gastroenterology 1978; 75: 623-31.

19 Yoshioka K, Keighley MRB. Anorectal myectomy for outlet obstruction. BrJ Surg 1987; 74: 373-6. 